\title{
Intralesional curettage versus Prosthetic replacement,which approach is suitable for bone tumors ?- A finite element analysis of limb salvage simulation in biomechanics
}

Matthew Jian-Qiao PENG

Guangzhou Medical University https://orcid.org/0000-0001-6518-6561

ChuLong SHEN

Foshan Hospital of Traditional Chinese Medicine

Hai-Yan CHEN

Hudong People's Hospital

Yong HU

University of Hong Kong

Er-Xing HE (D 13751857454@139.com )

YongQiang LAU

Foshan Hospital of Traditional Chinese Medicine

Research article

Keywords: Intralesional curettage; Prosthetic replacement; Bone tumor; Limb salvage; Simulation.

Posted Date: April 23rd, 2020

DOI: https://doi.org/10.21203/rs.3.rs-16685/v2

License: (c) (i) This work is licensed under a Creative Commons Attribution 4.0 International License. Read Full License 
The authors have withdrawn this preprint from Research Square 